\title{
ELT requirements for studies of galaxy formation/evolution and cosmology
}

\author{
Isobel M. Hook \\ Astrophysics, University of Oxford, U.K. \\ email: imh@astro.ox.ac.uk
}

\begin{abstract}
I summarise the ELT requirements derived from the science cases in the area of galaxy formation/evolution and cosmology. This summary is based on the talks, posters and discussion in parallel Session A of this meeting, but also makes use of various publically-available ELT science case documents. Topics covered include studies of black holes, dark matter, properties of galaxies at a range of redshifts (including the first galaxies to form), fundamental constants and cosmology.
\end{abstract}

Keywords. Galaxies: active, evolution, formation, high-redshift, Cosmology: miscellaneous, Instrumentation: adaptive optics, spectrographs

\section{Introduction}

The science cases discussed in this article are linked by the theme of understanding the formation of galaxies in the universe, and the nature of the universe itself. Topics covered include studies of black holes, dark matter, properties of galaxies at a range of redshifts (including the first galaxies to form), fundamental constants and cosmology. For each case I will briefly summarise the science case (many of which are described in more detail elsewhere in these proceedings) but will focus mainly on the requirements that these cases place on ELT design choices. In some cases a specific ELT instrument has been proposed to meet the science requirements and these will be summarised here also.

\section{Black Holes}

Only a few black holes have reliable mass measurements to date but these measurements pose some difficult questions. For example, why do their measured masses relate to the mass of their host galaxy bulges? This implies that the formation of black holes is linked to the formation of the galaxies themselves. In addition, the accretion history of AGN can be determined by comparing the cumulative mass function of quiescent galaxies locally with that of high- $z$ QSOs (see presentation by L. Ferrarese). Ideally we would like to extend mass measurements of black holes to much larger samples and over a wider range of redshifts. This implies increased sensitivity so that not only extremely massive black holes but also those in the intermediate mass range $\left(\sim 10^{6} M_{\odot}\right)$ can be measured.

\subsection{Requirements and implementation}

The main requirements are:

(a) spatial resolution to resolve the "sphere of influence" of the black hole,

(b) sensitivity to low surface brightness (particularly at high redshift) and

(c) spatial coverage to probe the dynamical structure in inner regions of galaxies - this is ideally suited to Integral Field Units (IFU) observations. 
The IRIS spectrometer, one of the instrument concepts for the $30 \mathrm{~m}$ Thirty Meter Telescope (TMT) project, (see presentation by D. Crampton) has been proposed to tackle this science case. The instrument concept consists of an AO-fed IFU with a $2^{\prime \prime}$ field of view (FoV). The TMT with Adaptive Optics (AO) can in principle resolve the sphere of influence of a $10^{6} M_{\odot}$ black hole at a distance of up to $24 \mathrm{Mpc}$ from us. Similarly a $10^{8} M_{\odot}$ black hole could be resolved to $z \sim 0.1$, a $10^{9} M_{\odot}$ black hole to $z=0.4$ and a $10^{10} M_{\odot}$ Black hole at all redshifts (where they exist!). A $100 \mathrm{~m}$ telescope could in principle resolve $\sim 1$ order of magnitude lower in mass at a given distance than a $30 \mathrm{~m}$ telescope (see the OPTICON ELT science case book, Hook (Ed.) 2005). In practice these measurements will be limited by surface brightness sensitivity - thus this is a driver for as large a telescope as possible not just for resolution but also for collecting area.

\section{Dark Matter in Ellipticals}

Although dark-matter dominated theories of galaxy formation have been successful in many ways at explaining the growth of large scale structure and the evolution of galaxies, some problems have emerged when numerical simulations are confronted with detailed observations. Examples include inconsistencies in the number of satellites galaxies within galaxy haloes and the density profiles in galaxy cores.

Whereas for spiral galaxies HI provides a convenient tracer beyond the edge of the light profile, for ellipticals the use of "test particles" is required to explore the dark halo (see presentation by P. Côté, and Côté et al. 2003). Suitable test particles include planetary nebulae (PNe) and globular clusters (GCs).

To study the dynamics and distribution of dark matter one would like to measure mass profiles and the distribution of orbits for a large sample of galaxies. Ideally 1000 GCs and PNe per galaxy would be observed. With current telescopes only 4 galaxies have sufficient numbers of observable GCs and PNe to be studied in this way. With a $30 \mathrm{~m}$ telescope such as TMT, about 50 galaxies will be within range.

\subsection{Requirements and implementation}

The science goals require measurements of velocities to an accuracy of $20 \mathrm{~km} \mathrm{~s}^{-1}$. Observations are expected to take $\sim 1 \mathrm{hr}$ per system on a $30 \mathrm{~m}$ telescope - all 50 available galaxies could be observed in 2-3 weeks provided the instrument used has a high multiplex capability $(\sim 500)$ to allow many "test particles" to be observed simultaneously. The targets would need to be selected beforehand from 8-10m telescope data.

This is one of 5 key science cases for the WFOS instrument concept for TMT. At least initially, this instrument would operate in natural seeing (with a slit width $=0.75^{\prime \prime}$ ). WFOS is proposed to have a FoV of $80 \mathrm{sq}$ arcmin. For this program it would be used with spectral resolution of $\mathrm{R}=2000$ in the blue arm $(0.47-0.54 \mu \mathrm{m})$ and $\mathrm{R}=5000$ with the red arm $(0.83-0.88 \mu \mathrm{m})$.

\section{Evolution of galaxies: physics of galaxies with $1<z<5$}

Closely linked to the previous case, a major goal in the understanding of formation of galaxies is to better understand the physical processes involved, particularly feedback processes such as the effects of supernovae and AGN on surrounding star formation.

To measure the effects of these processes requires spatially resolved (on kpc scales) spectroscopic studies of large samples of galaxies over the period when galaxies were forming the bulk of their stars and evolving $(1<z<5)$. The key parameters that can be derived 
from such measurements are the star formation history, stellar mass, extinction, metallicity, ionisation state, line shapes (which provide information on outflows and winds) and internal dynamics (which provide dynamical mass measurements). These measurements could then be related to the measurements of build-up of dark matter in galaxy haloes provided by dynamical measurements of satellites and lensing measurements at a range of redshifts.

\subsection{Requirements and implementation}

This science case has been discussed in several ELT science documents. For example in the OPTICON European science case it was suggested that an instrument similar to the FALCON concept (Hammer et al. 2004) would be suitable. This concept has multiple IFUs patrolling a large FoV $\left(2^{\prime}\right.$, with a goal of $\left.10^{\prime}\right)$, with each IFU fed by local AO correction (i.e. the multi-object AO concept) providing 50-100 mas images. The IFUs could be of two different types with different pixel scales and FoVs. Spectral resolution of $\sim 5000-10000$ is required over the wavelength range 0.7 (or preferably 0.3 ) to $2.5 \mu \mathrm{m}$ to resolve key diagnostic spectral features. This particular concept was considered for a $100 \mathrm{~m}$-class telescope, where exposures would be of the order of 1 night per field. A 30m telescope could also make great advances although in less detail - for example integrated properties could be measured (metallicity, ages, winds) to $z \sim 3$.

\section{The First Galaxies}

At higher redshifts $(z>6)$, where galaxies are believed to be assembling, there is considerable uncertainty about the source properties. Extrapolations must be made from existing high- $z$ galaxy samples (see talks by J. Bergeron and J.-G. Cuby). One such sample, from the Subaru-XMM Deep Survey, contains sufficiently large numbers of sources to allow studies of the their properties and clustering in the range $3<z<6$ (see presentation by K. Sekiguchi). Although very deep, $\left(\mathrm{B}_{\mathrm{AB}}=28.2, \mathrm{~K}_{\mathrm{AB}}=24.9\right)$, even deeper surveys than this will be needed to reach $z>7$ and to probe normal $L^{*}$ galaxies above $z=3$. The next step is to obtain spectroscopy of both UV continuum and emission lines for galaxies with $z=7-15$. A large survey would be required to measure properties of the stellar populations, metallicity and masses for a large sample at high redshift.

\subsection{Requirements and implementation}

Extrapolation of existing high-redshift galaxy luminosity functions give estimated source densities between a few times 0.01 and 1 per square arcmin at $z>6$ and a few tenths at $z \sim 7-8$ to a depth of $A B=28.5$ (the approximate limit for spectroscopy with a $100 \mathrm{~m}$ telescope). This rarity of objects drives requirements to large (patrol) fields of view (preferably of order $10^{\prime}$ or even larger) because of the rarity of sources to enable efficient multiplexing.

Another important driver is the size of the sources at high redshift. Extrapolating the size-redshift relation seen between $z \sim 2$ and $z \sim 6$ (Bouwens et al. 2004) it is estimated that the half light radius is 170 mas at $z=6$ and 150 mas at $z=10$. There is little information on the substructure in galaxies at these redshifts but to match best estimates (e.g. from lensed high-redshift galaxies) the objects should be sampled at the $\sim 10-30$ mas level (see presentation by J.-G. Cuby). Note that this is lower resolution than the theoretical diffraction limit for an ELT $(\sim 8$ mas and $\sim 3$ mas for 30 and 100m telescopes respectively at 1 micron).

Finding such sources may require imaging by space-based telescopes (such as JWST) or could possibly be carried out from the ground by ELTs themselves using wide-field 
imagers with "moderate" AO correction. The minimum size of telescope required for an imaging search is to be determined.

For spectroscopic confirmation of such faint targets (AB 28) large, ground-based telescopes are required. Note that despite higher background from the ground, the collecting area and resolution of ELTs mean they will have comparable or better performance than JWST in spectroscopic modes in the near-infrared. This science case requires good sky coverage - not only "random" fields - because of the desire to observe fields that have been studied with other facilities (e.g. at other wavelengths).

A possible implementation of these requirements is the MOMFIS instrument concept for the OWL telescope (see talks by J.-G. Cuby and P. Bouchet). This instrument would have $R>4000$ for $\mathrm{OH}$ suppression over the wavelength range $1.0-2.4 \mu \mathrm{m}$. Thirty IFU pickoffs patrol a FoV of $5^{\prime} \times 5^{\prime}$. The IFUs have individual FoVs of 0.5 arcsec and sampling of 10-20mas. Multi-Object AO (local correction) would provide images of about 20-50mas (simulations show that GLAO would not meet the requirements - see presentation by P.Bouchet). Note also that the sky coverage with natural guide stars is only marginally acceptable so a laser guide star system is preferred.

\section{High Redshift Supernovae}

Type Ia supernovae ( $\mathrm{SNe}$ ) can be used as distance indicators for measurement of cosmological parameters and to probe the effects of "Dark Energy", while Type-II SNe can be used as direct tracers of the star formation history of the Universe.

The OPTICON ELT science case includes a simulated Hubble diagram for supernovae of different types (see also Della Valle et al. 2004) and shows that a 100m telescope could in principle detect and obtain spectroscopic redshift and supernova Type determinations for Type Ia supernovae to $z \sim 4$, and core-collapse SNe to $z \sim 10$. ELTs of all sizes would benefit this area of research. A $20 \mathrm{~m}$ class telescope could observe Type Ia SNe beyond the current limit of $z \sim 1.2$, and could also be crucial in studying systematic effects (for example signs of evolution in the SNIa population could potentially be observed using detailed spectroscopy).

\subsection{Requirements and implementation}

Being point sources, SNe are one class of source at cosmological distances that benefit from observations with high spatial resolution (they are in the regime where observing time scales inversely as $\mathrm{D}^{4}$, where $\mathrm{D}$ is the telescope diameter).

Finding the supernovae will be a challenge with ELTs. To get the highest signal-tonoise and a good probability of finding multiple SNe in a field requires fairly high Strehl $\mathrm{AO}$ correction over a large FoV. The possible implementation discussed in the OPTICON science case calls for an MCAO-fed infrared imager on OWL (100m telescope) with a $2^{\prime} \times 2^{\prime} \mathrm{FoV}$ (requiring a very large number of detector pixels!). Spectroscopic follow-up requires spectral resolution of $R \sim 2000$. The SNe could be observed spectroscopically one at a time, or if a patrol FoV of $5^{\prime}$ or larger were available then multiple targets could be observed simultaneously.

\section{Cosmic Expansion}

An interesting application for ELTs is the possibility of directly measuring the dynamics of the expansion of the universe (see talk by L. Pasquini). The method involves measurement of the very small shift in the wavelength of absorption lines in QSO spectra caused by the expansion of the universe over a $\sim 10$ year period. Being a dynamical 
measurement it provides a fundamental test of the predictions of General Relativity, specifically whether the dynamical expansion of the universe matches the geometrical expansion measured by Type Ia supernovae and other methods.

\subsection{Requirements and implementation}

Since the effect is very small (a few cm/s/yr, Sandage 1962) this experiment requires very high resolution spectroscopy and places extreme requirements on stability and calibration accuracy of the spectrograph. A further crucial requirement is very high signal to noise in order to measure line centres to the required precision. Thus an ELT is required to obtain sufficient photons for the measurement. The implementation of this experiment has been studied as one of the OWL instrumentation studies - the CODEX instrument concept has a spectral resolution of $R \sim 150000$ and would operate over $0.4-0.6 \mu \mathrm{m}$. The QSOs would be observed one at a time under natural seeing conditions.

\section{Fundamental constants}

A second application of very high-resolution spectroscopy on ELTs is to place constraints on the variation of the fine structure constant $(\alpha$, see talk by P. Molaro and poster by Levshakov). The implications for detection of a varying $\alpha$ are profound - it may imply the existence of extra spatial dimensions, or a scalar field acting late in the Universe. Current measurements of QSO absorption lines by two groups using 8-10m telescopes give two conflicting results (one team claiming detection of a varying $\alpha$ whereas the other finds no significant variation).

\subsection{Requirements and implementation}

To make a significant advance over the current measurements requires resolving even the sharpest absorption lines, of order $1 \mathrm{~km} \mathrm{~s}^{-1}$ wide. An ELT could provide significant advance in this field. For example a $60-100 \mathrm{~m}$ ELT would give 2 (or perhaps more) orders of magnitude improvement in precision. This experiment has been considered as part of the CODEX study summarised above, and the requirements are similar.

\section{Requirements for follow up of X-ray surveys}

An important use of ELTs will be follow up of sources discovered in other wave-bands. Spectroscopy of such sources will provide redshifts, and a better understanding of the physical nature of the sources. At present about 30\% of sources from deep X-ray surveys do not have spectroscopic redshifts (their identifications are fainter than $\mathrm{i}=25$ - see talk by R. Griffiths). Indeed even some sources discovered by HST in optical wavelengths are beyond the reach of spectroscopy with $8 \mathrm{~m}$ class telescopes today.

New facilities being planned such as XEUS and Constellation-X missions at X-ray wavelengths, SPICA (mid-IR), JWST (near-IR), ALMA (sub-mm) and SKA (radio) in the future will provide even fainter sources requiring spectroscopy. For example XEUS will reach at least an order of magnitude fainter in X-ray flux and optical identifications are likely to have magnitudes pushing $I=30$.

XEUS will have a FoV of $\sim 7$ arcmin, therefore an ELT FoV of similar size would be well matched for efficient multi-object spectroscopy. However the spatial resolution of XEUS will be of order $\sim 5^{\prime \prime}$ only, so finding the location of the sources will be a problem. 
Table 1. Summary of Science requirements. Entries in brackets are goals rather than absolute requirements. The entries in italics were taken from the OPTICON ELT science case (Hook (Ed) 2005) and were not derived from presentations at this meeting.

\begin{tabular}{|c|c|c|c|c|c|}
\hline $\begin{array}{l}\text { Science } \\
\text { Case }\end{array}$ & FoV & $\begin{array}{l}\text { Spatial } \\
\text { resolution }\end{array}$ & $\begin{array}{l}\text { Spectral } \\
\text { resolution }\end{array}$ & $\begin{array}{l}\lambda \\
(\mu \mathrm{m})\end{array}$ & Comments \\
\hline Black Holes & $\begin{array}{l}2^{\prime \prime} \\
\left.\text { (goal } 5^{\prime \prime}\right)\end{array}$ & $\begin{array}{l}\text { Diffraction } \\
\text { limited }\end{array}$ & Few x 1000 & $\begin{array}{l}1 \mu \mathrm{m} \\
\text { or optical }\end{array}$ & IFU ideal \\
\hline $\begin{array}{l}\text { Dark matter in } \\
\text { Ellipticals }\end{array}$ & $9^{\prime} \times 9^{\prime}$ & $\begin{array}{l}\text { Natural } \\
\text { seeing }\end{array}$ & $\begin{array}{l}2000(\mathrm{~B}) \\
5000(\mathrm{R})\end{array}$ & $0.5-0.9$ & $\begin{array}{l}\text { Multiplex } \\
\sim 700\end{array}$ \\
\hline First galaxies & $\begin{array}{l}5^{\prime} \times 5^{\prime} \\
\left(\text { goal } 10^{\prime} \times 10^{\prime}\right)\end{array}$ & $20-50$ mas & 2000 & $1.0-2.4$ & $\begin{array}{l}\text { Multi-IFU } \\
\text { (or slits?) }\end{array}$ \\
\hline$z=1-5$ galaxies & $\begin{array}{l}5^{\prime} \times 5^{\prime} \\
\left(\text { goal } 20^{\prime} \times 20^{\prime}\right)\end{array}$ & $10-50$ mas & $5000-10000$ & $\begin{array}{l}0.7-2.5 \\
(0.3-2.5)\end{array}$ & Multi-IFU \\
\hline $\operatorname{CODEX} / \Delta \alpha$ & $\begin{array}{l}1^{\prime \prime} \\
\text { (single sources) }\end{array}$ & $\begin{array}{l}\text { Natural } \\
\text { seeing }\end{array}$ & $\begin{array}{l}150,000 \\
(300000)\end{array}$ & $0.4-0.6$ & $\mathrm{~cm} / \mathrm{s} / \mathrm{yr}$ stability \\
\hline X-ray follow-up & $7^{\prime} \times 7^{\prime}$ & & & & $\begin{array}{l}\text { Target } \\
\text { location? }\end{array}$ \\
\hline Supernovae & $2^{\prime} \times 2^{\prime}$ & $\begin{array}{l}\text { Strehl }(\mathrm{K}) \\
=0.5\end{array}$ & 2000 & $1.0-2.5$ & $\begin{array}{l}\text { imaging \& } \\
\text { (MOS?) spectroscopy }\end{array}$ \\
\hline
\end{tabular}

\section{Requirements Summary}

Table 1 summarises the requirements from the various science cases discussed in this paper.

- Spatial resolution: In some science cases high-Strehl adaptive optics is desired, namely the cases involving point sources such as supernovae, and studies of Black Holes. However as can be seen from Table 1, there are several cases that can be done in natural seeing or with "moderate" seeing improvement, i.e. they do not require the ELT to operate at its diffraction limit. There is however some discussion as to whether wide-field AO systems such as GLAO or MOAO will provide seeing improvement in the sense of a globally smaller PSF, or whether they will provide a PSF with a small amount of energy in a diffraction-limited core, and with an uncorrected halo of similar extent to the natural seeing disk. While the latter would be some improvement over natural seeing, a diffraction-limited core is in some ways overkill when what is really needed is to reduce the PSF halo component to roughly match the size of the sources. The effect of AO on high-redshift galaxy observations needs to be studied with simulations using realistic AO PSFs. Clearly we will learn more about wide-field AO performance from systems that are being planned and implemented now on $8 \mathrm{~m}$ telescopes.

- Field of View (FoV): Large Scale Structure at all redshifts pushes the FoV of future telescopes to large FoVs in order to overcome cosmic variance in galaxy surveys. However the difficulties in building ELTs with large FoVs mean that $8 \mathrm{~m}$ telescope with wide fields of view (1 degree across and larger) could be very competitive in this domain in terms of the time taken to cover wide areas. Scaling relations as a function of telescope diameter have been calculated for various regimes (see talk by M. Colless). For galaxies there may be gains scaling as $D^{4}$ for medium-resolution $(R>4000)$ wide field spectroscopy only if AO can provide $\sim 0.1^{\prime \prime}$ images over wide fields.

- Spectral resolution: Many of the cases above require moderate resolution (a few thousand to 10000$)$ to probe the physical processes in high-redshift galaxies. In some 
cases only low resolution is needed (for example to measure redshifts) but in oder to suppress $\mathrm{OH}$ lines in the infra-red, the instrumentation may need to have $R>3000$. Very high resolution is needed for QSO absorption line studies, particularly those that involve measuring narrow metal lines (see the CODEX cases).

- Wavelength: The majority of cases discussed in this session require observations in the near-infrared $(1-2.4 \mu \mathrm{m})$ with some extending into the optical. No cases were presented during this session for mid-IR observations although these wavelengths would be useful for studying obscured AGN and ultra-luminous infrared galaxies at high redshift (e.g. see the science case for T-OWL, Lenzen et al. 2005). The case has also been made for a sub-mm camera on OWL (named "SCOWL", Holland et al. 2003) which would provide a fast sub-mm survey capability for finding high redshift galaxies. If the mid-infrared and sub-mm cases are considered high-priority then this will have strong implications for the choice of site.

\subsection{Requirement Priorities}

To summarise, the principal requirements derived from session A are (in my opinion) large telescope aperture and large FoV (usually meaning a patrol field for multiple pickoffs rather than a fully instrumented or AO corrected field of view). Another important factor is good sky coverage (implying the use of laser AO). In many cases spatial resolution is not a major driver but enhancements compared to natural seeing are desirable in order to increase contrast of faint sources.

Exceptions to the above are very high resolution QSO absorption line studies (see the CODEX cases) which do not require a large FoV as sources would be observed one at a time, the Black Holes case (which needs the highest resolution over small FoV) and the high-redshift supernova case which, because the targets are point sources, would benefit from high-Strehl images, preferably over a reasonably large FoV.

\section{Conclusions and Discussion}

Below I summarise some questions that arose from the discussion without attempting to answer them.

- what is the practical limit of FoV/ telescope aperture? Are there natural strategies to tackle these scientific goals using more than one ELT with different optimisation? For example one could imagine using a "nested" survey strategy (e.g. see talk by P. McCarthy) where moderately high- $z$ galaxies $(z>5)$ are observed with a wide-field 20$30 \mathrm{~m}, z>7$ galaxies with an AO-optimised $20-50 \mathrm{~m}$ and the highest $\mathrm{z}$ galaxies $(z>10)$ with the largest ELTs available ( $>50 \mathrm{~m})$.

- Are there any natural break points between $D^{2}$ and $D^{4}$ science?

- Can the focal plane be instrumented such that many science projects can be carried out simultaneously?

- Should we consider coordination between telescope projects from a very early stage? (for example we could consider coordination of first light instruments, and build timesharing into the operations model from the start).

\section{Acknowledgements}

I would like to thank all the speakers, poster authors and all those who participated in the discussion during the galaxy formation/evolution and cosmology session. 


\section{References}

Lenzen, R., Brandl, B. et al. 2005, OWL Instrument Concept Study for T-OWL, ESO.

Bouwens, R. et al. 2004, ApJ 611, L1

Côté, P., McLaughlin, D.E., Cohen, J.G., Blakeslee, J.P. 2003, ApJ 591, 850

Della Valle, M., Gilmozzi, R., Panagia, N., Bergeron, J., Madau, P., Spyromilio, J., Dierickx, P. 2005, in: A. Lobanov (ed.), Exploring the Cosmic Frontier, Springer-Verlag series "ESO Astrophysics Symposia", in press. See also astro-ph/0504103

Hammer, F. et al. 2004, SPIE 5382, 727

Holland, W. et al. 2003, SPIE 4840, 340

Hook, I.M. (ed.), 2005, The Science Case for the European 50-100m telescope, OPTICON. See http://www.astro-opticon.org/networking/elt.html

Sandage, A. 1962, ApJ 136, 319

\section{Discussion}

DEnNEFELD: You mentioned that SNe (and black holes) were the only cases in this session where 'single point source' operation was needed. Would it not be preferable to use 'single IFU' instead, because you may want to study simultaneously the underlying galaxy, and need to subtract its spectrum anyhow to get a clean SN spectrum?

Hook: Yes that is correct - both these cases would benefit from spatially-resolved spectroscopy. The main point I was trying to make about SNe is about the requirement on image quality delivered to the instruments. SNe, being point sources, would benefit from 'full' AO such as MCAO.

Gilmore: To what extent is the support for studying faint galaxies with angular resolution no less than 0.1 arcsec a consequence of our lack of a higher resolution image of any of these sources?

Hook: For the most distant galaxies $(z>10)$ the main issue is sensitivity - getting all the light from the object down the 'slit' is the main goal, and there probably wont be enough flux to do highly spatially resolved spectroscopy. At lower redshifts spectroscopy on spatial scales of 20-50 mas should be possible. Depending on the nature of these sources smaller scales may indeed be better, e.g., if there are spatially unresolved 'knots'. So perhaps if JWST or ELTs themselves provide images showing smaller features then we might want higher spatial resolution spectroscopy. The best limits we have at present come from a gravitationally lensed high- $z$ galaxy which has been magnified so that substructure is visible. The substructures seen in the reconstructed de-warped image were used to set the pixel size for the MOMFIS IFUs, for example. 